\title{
Host Feasibility Investigation to Improve Robustness in Hybrid DWT+SVD Based Image Watermarking Schemes
}

\author{
Tanya Koohpayeh Araghi ${ }_{1},{ }^{1,2}$ Azizah Abd Manaf, ${ }^{3}$ Ala Alarood, ${ }^{3}$ and Azida Binti Zainol ${ }^{3}$ \\ ${ }^{1}$ Advanced Informatics School Universiti Teknologi Malaysia, 54100, Kuala lumpur, Malaysia \\ ${ }^{2}$ Iranian Social Security Organization, 3817793164, Arak, Iran \\ ${ }^{3}$ Department of Computer Science, Faculty of Computing and Information Technology, University of Jeddah, 21432, \\ Raids, Saudi Arabia
}

Correspondence should be addressed to Tanya Koohpayeh Araghi; tanya.koohpayeh@gmail.com

Received 13 April 2018; Revised 12 August 2018; Accepted 14 August 2018; Published 10 October 2018

Academic Editor: Alexander Loui

Copyright (C) 2018 Tanya Koohpayeh Araghi et al. This is an open access article distributed under the Creative Commons Attribution License, which permits unrestricted use, distribution, and reproduction in any medium, provided the original work is properly cited.

Today, we face different approaches to enhance the robustness of image watermarking schemes. Some of them can be implemented, but others in spite of spending money, energy, and time for programming purpose would fail because of not having a strong feasibility study plan before implementation. In this paper, we try to show a rational feasibility study before implementation of an image watermarking scheme. We develop our feasibility study by proposing three types of theoretical, mathematical, and experimental deductions. Based on the theoretical deduction, it is concluded that the "S" coefficients in the second level of Singular Value Decomposition (SVD) offer high robustness to embed watermarks. To prove this, a mathematical deduction composed of two parts is presented and the same results were achieved. Finally, for experimental deduction, 60 different host images in both normal and medical images from various sizes of $256 * 256$ to $1024 * 1024$ were imposed to 9 common geometric and signal processing attacks and the resistances of "S" coefficients against the attacks in the first and second levels of SVD were compared. Experimental result shows significant enhancement in stability and robustness of the " $\mathrm{S}$ " coefficients in the second level of SVD in comparison to the first level. Consequently all theoretical, mathematical, and experimental deductions confirmed domination of the " $\mathrm{S}$ " coefficients in the second level of SVD than the first level. In this paper, we do not show any specific implementation for the watermarking scheme. Instead, we investigate the potential performance gains from the singular values (S), of the second level of SVD and Discrete Wavelet Transform (DWT), and prove their superiority in comparison to conventional SVD+DWT watermarking schemes.

\section{Introduction}

Digital Image watermarking is introduced to protect the digital medium from illegitimate access and illegal alteration [1-3]. To achieve the required functionalities in the target application special care has to be taken so that the embedded watermark can resist attacks and manipulations [4]. Various techniques are introduced in digital image watermarking. In [5] different techniques of image watermarking are divided into spatial and transform domain and it is mentioned that the transform domain techniques provide higher robustness and imperceptibility than spatial domains to embed the watermark images. On the other hand in [6] among different transform domain techniques, DWT was referenced as a superior transform domain technique for image watermarking while combination of this technique with other transform domains can compensate the flaws of using each technique solitary. In [7-11] hybridization of DWT and SVD is considered as an efficient combination to increase the resistance of the watermarking scheme against signal processing and noise attacks.

However, depending on attack's types and intensity this hybrid technique is not robust as well.

In this paper, we purely investigate host images to find regions of interests representing the least distortion against geometric and signal processing attacks after Discrete Wavelet Transform (DWT) and Singular Value Decomposition (SVD) transformations. We called this investigation as a feasibility study in order to find the regions of interest and consequently to improve robustness. The aim is to prove 
an idea of increasing the robustness of hybrid DWT+SVD schemes by selecting the regions of interest in the host image.

In other words the ROIs are selected basically by analyzing the host image and investigating the theories ending up to high robustness.

For this purpose, a feasibility study is developed in three phases. In the first phase, the theoretical deduction supporting basic idea of dominance of " $\mathrm{S}$ " coefficients in the second level of SVD that can enhance robustness is investigated. In the second phase, mathematical deductions to prove the dominance of these areas are developed and, finally, the hospitability of the candidate areas for embedding the watermark is investigated by exposing the host images on 9 types of geometric and signal processing attacks. This paper is organized as follows: first, a description about the SVD and DWT and the structure of an image when it is decomposed by SVD and the method to find ROIs is presented. Secondly, the theoretical deductions and preliminaries to enhance image resistance geometrical and noise attacks are described. In the third phase, this theoretical deduction is proved mathematically and finally in order to demonstrate the superiority of the "S" coefficients in the second level of SVD, a wide range of medical and normal images is exposed on 9 most common types of image processing and geometric attacks and stability of " $\mathrm{S}$ " coefficients in the first and the second level of SVD are compared to prove both phases of theoretical and mathematical deductions experimentally.

As it is mentioned before, there is no specific implementation in this paper. In fact, we state the way that we approached to the idea of stability of the " $\mathrm{S}$ " coefficients against attacks and consequently selected as the regions of interests. In this paper, we just state an approach tending to ensure an economic implementation without wasting time, money, and energy. The whole implementation of the proposed watermarking process is represented in [12].

\section{An Overview on SVD and the Proposed ROIs}

Singular value decomposition (SVD), decomposes a matrix into left and right singular vectors and a diagonal matrix of singular values. If $\mathrm{X}$ would be an $\mathrm{m} * \mathrm{n}$ matrix, it can be written as $\mathrm{X}=\mathrm{USV}^{\mathrm{T}}$, where " $\mathrm{U}$ " is an orthogonal $\mathrm{n} * \mathrm{n}$, " $\mathrm{V}$ " is an $\mathrm{m} * \mathrm{~m}$ orthogonal matrix and, and " $\mathrm{S}$ " is a $\mathrm{n} * \mathrm{~m}$ matrix such that its first $r$ diagonal entries are nonzero singular values of $\partial 1, \partial 2, \partial 3, \ldots, \partial \mathrm{r}$ and the rest of entries are all zero. The order of values in singular values is $\partial 1>\partial 2>\partial 3>\ldots>$ $\partial \mathrm{r}$. The expression of $\mathrm{X}=\mathrm{USV}^{\mathrm{T}}$ is called the singular value decomposition for $\mathrm{X}$. This definition is shown below and in Figure 1.

$$
\text { Matrix X before SVD decomposition: } X=\left[\begin{array}{ccc}
\mathrm{X}_{11} & \cdots & \mathrm{X}_{1 t} \\
\vdots & \ddots & \vdots \\
\mathrm{X}_{m 1} & \cdots & \mathrm{X}_{m t}
\end{array}\right]
$$

Regarding an image as a matrix, it can be decomposed by $\mathrm{U}$ and $\mathrm{V}$ singular vectors and $\mathrm{S}$ singular values. In image decomposition by SVD, U and V carry the whole geometric

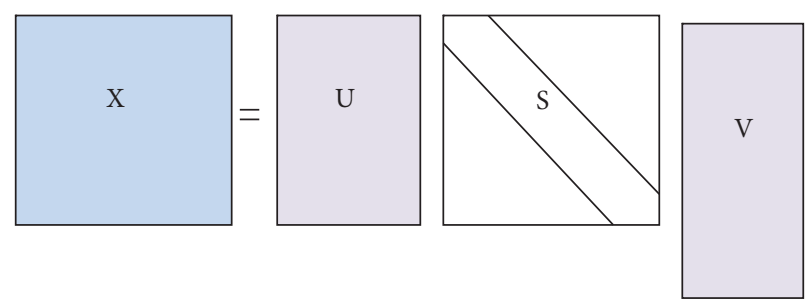

FIGURE 1: Matrix X after SVD decomposition.

specifications of the image while the luminance is carried by $S$. Since the $S$ coefficients are geometric-free, they are less affected by geometric attacks like rotation and scaling. However, they still cannot resist several geometric and a range of signal processing attacks $[8,14-16]$. In this research the aim is to find regions from $S$ coefficients that not only resist geometric but also are less affected by signal processing changes.

To find the regions of the host image with high resistance against distortion, we need to find the highest energetic parts of image after SVD transform. Since in S coefficients the highest energetic portion is located in $S(1,1)$, the image blocking can be a good idea.

After one level DWT, the host image is divided into $\mathrm{n} * \mathrm{n}$ blocks while $\mathrm{n}<$ watermark size. $\mathrm{S}(1,1)$ from each block is collected in a new matrix, and then the second level of SVD is imposed on this matrix. The $\mathrm{S}$ coefficients of this matrix are considered as the ROIs and we prove that these secondary coefficients are more robust and stable than $\mathrm{S}$ coefficients of the first level of SVD after one level DWT. Figure 2 shows the process in detail.

The scheme can be performed for every size of $n * n$ host image and it depends on the size of the chosen watermark image. For example, in a $64 * 64$ watermark image and one level of DWT applied on it, before embedding to the host image, the size of the watermark becomes $32 * 32$. Hence, we need $32 * 32$ blocks to hide the watermark. Considering that the host image size is $512 * 512$, after one level DWT it is changed to $256 * 256$ and for receiving $32 * 32$ blocks we need to divide $256 * 256$ into $8 * 8$ blocks to get $32 * 32$ places for inserting the watermark. As a result, the optimum block size is selected as $8 * 8$; for other sizes of the cover images the same calculations need to be performed.

The rest of the paper is assigned to prove the predominant of the S coefficients in the second level of SVD compared with their peers in the SVD level one, as a desire host for information hiding. In the following, three phases of our deductions constitute theoretical, mathematical, and experimental deductions presented.

\section{Theoretical Host Feasibility Deduction}

It is necessary to check the host image in order to determine whether the selected points (regions of interests) are suitable places to embed watermark or not. Suitability is defined in terms of stability against changes or robustness against attacks and imperceptibility or invisibility based on human 


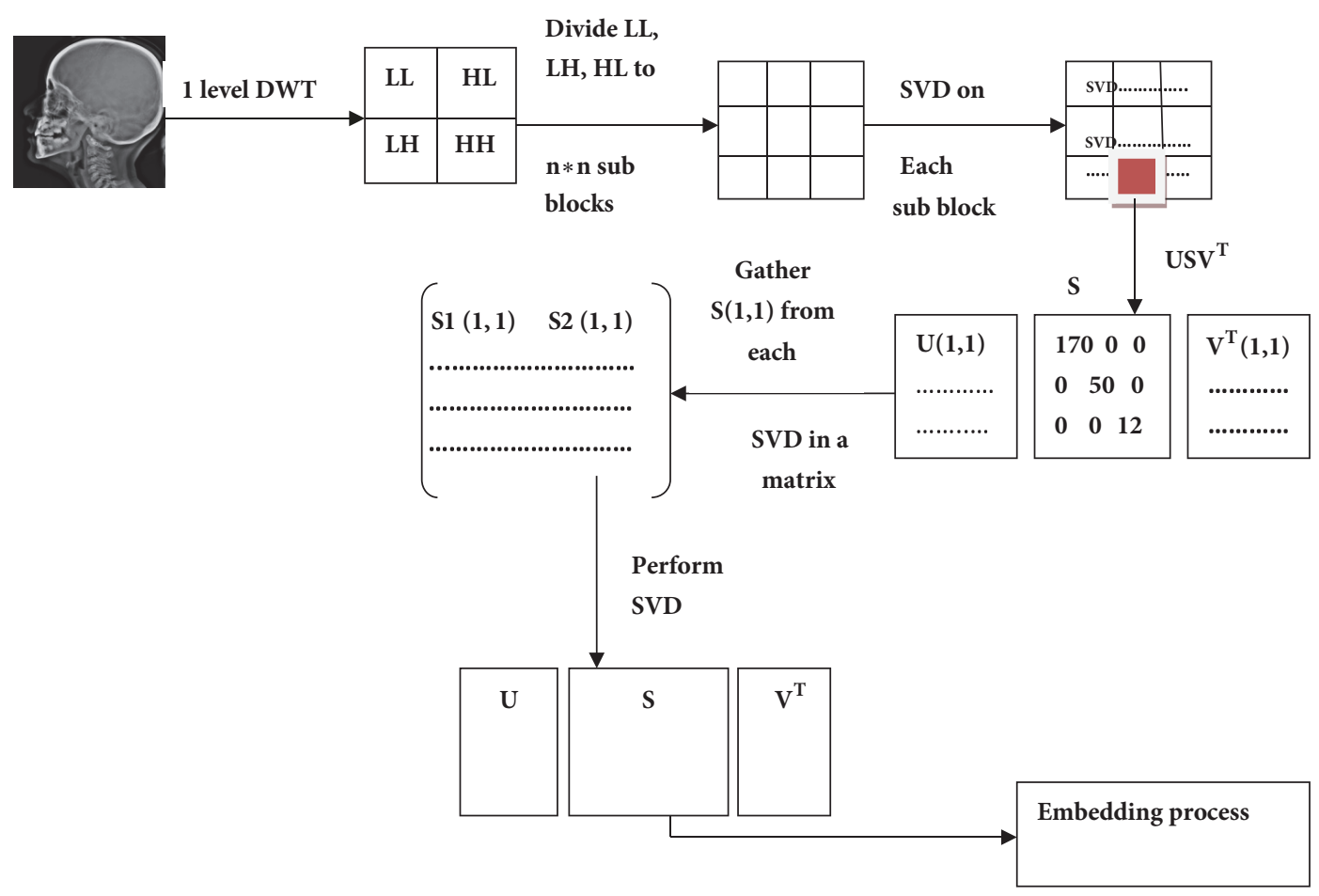

FIGURE 2: Selected "S" coefficients of the second level of SVD.

visual system [17]. In this section, we try to find stable places in the host image such that their stability can be justified theoretically. In other words, we have to find proficient points among singular values or vectors of SVD. These points will be selected based on the facts and theories presented about robustness in SVD and DWT (as another transform domain to link with SVD to make a hybrid robust scheme). Theoretically the following deductions lead to "S" coefficients in the second level of SVD as selected regions of interest.

3.1. Basic Ideas and Theoretical Deduction to Enhance Robustness Respecting to Imperceptibility. In the following, basic ideas to achieve robustness are explained.

3.1.1. Scattering. According to [18], to make the maximum robustness against attacks, the watermark should be spread all over the entire cover image instead of only being hidden in a limited number of bits. Such scheme ensures the robustness of the watermark against attacks.

Since SVD decomposes the image matrix to its constituent elements as singular values and left and right singular vectors, a greater degree of spreadness will be achieved by executing this transform technique. DWT also performs a multiresolution technique in which the image can be defined based on each frequency subband [5]. Thus, the watermark image can be added to each subband frequency as a small noise. Adding the watermark as small noise to high frequency subbands is becoming recognizable by human visual system (HVS), because they have smaller values. In contrast, low frequency sub band includes higher values so that the effect of changes can rarely be seen in them. Figure 3 shows the values of an image after one level wavelet decomposition in each frequency sub band and how adding a small noise after DWT is scattered in the whole of image by checking the values.

3.1.2. Separability. According to definition of [13], separability is the difference between the correlation of watermark and the highest correlation of the watermark after attack. Higher separability leads to higher robustness. It is referred to a parameter " $S$ " as separability to measure the robustness.

$$
\mathrm{S}=\min \left(\mathrm{C}-\mathrm{C}_{\mathrm{K}}\right)
$$

where "C" is regarded as the correlation between the embedded watermark and the most prominent coefficient of the attacked watermarked and "CK" is the correlation between Kth random

Watermarked and the most prominent coefficient of the watermarked data after attack as shown in Figure 4. The more separability, the more robust scheme will be offered.

In other words, the watermark image should be hidden to the most prominent selected coefficient of the cover image, so that less effect can be imposed by attacks.

To obey this matter and also to scatter the watermark, the cover image will be transformed by DWT, and selected sub bands divided to $\mathrm{n} * \mathrm{n}$ non overlapped blocks ( $\mathrm{n}$ is a power of 2). Then SVD transform will be performed for each block. Since now, spreading the watermark is performed. In the second step we have to find the most prominent coefficients to hide the watermark. According to the SVD definition, singular values are less affected by geometric and 


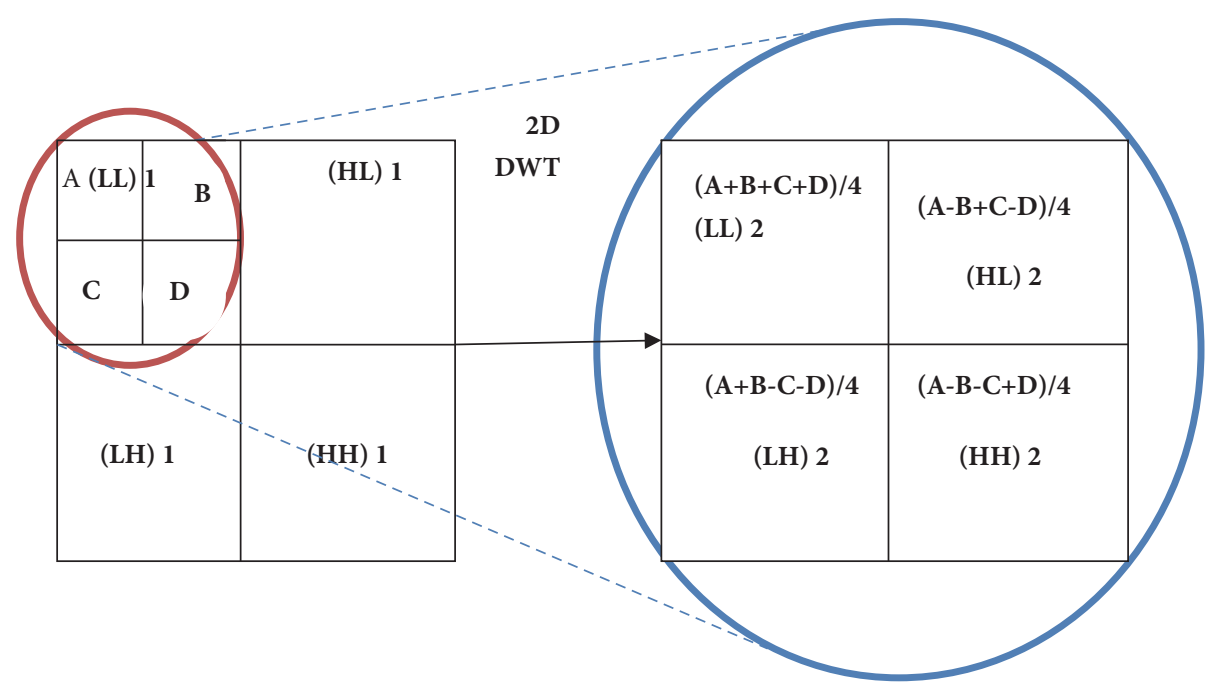

FIGURE 3: New values of an image after one level DWT decomposition.

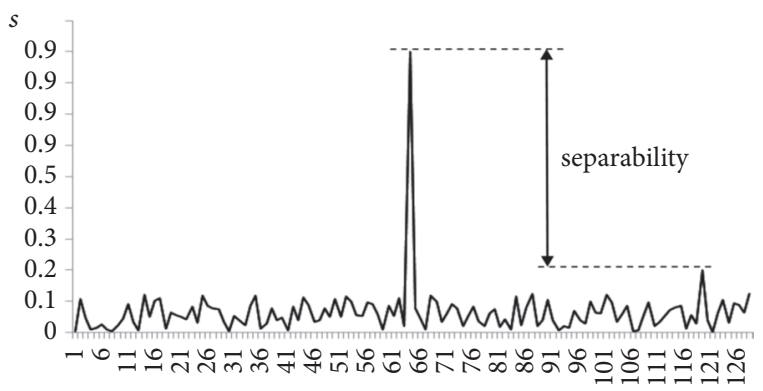

FIgURE 4: Separability in a Robust Watermarking [13].

signal processing attacks, and among singular values, those at the coordinates of $(1,1)$ have the most energy compaction. Equation (3) displays the order of singular values of a matrix:

$$
\left(\begin{array}{cccc}
1250 & 0 & 0 & 0 \\
0 & 500 & 0 & 0 \\
0 & 0 & 75 & 0 \\
0 & 0 & 0 & 3
\end{array}\right)
$$

As it is shown in Equation (3), singular values at the $(1,1)$ position are larger than the other singular values. Hence, the first singular values at the mentioned position will be chosen and kept in an ultimate matrix. This matrix is built from the all singular values of each $\mathrm{n} * \mathrm{n}$ block at $(1,1)$ position. Thus, it includes the highest energy compaction and is less influenced by synchronization. In the next step, the SVD transform will be performed for the second time. The singular values produced by this transformation also inherit the dominant characteristics of the last level.

3.1.3. Stability of SVD Singular Values. After SVD transform, the singular values of an image matrix are invariant to transpose, flip, rotation, scale, and translation $[19,20]$. This means that after mentioned attacks, the singular values are less affected by them. Then, they can be good candidates for embedding the watermark image. It is expected that these characteristics will be inherited by each level of SVD decomposition. Thus, it can be concluded that the singular values at higher level of SVD would be more robust to geometric and signal processing attacks.

\section{Mathematical Host Feasibility Deduction}

In order to prove the robustness of the selected areas which are the "S" coefficients of the second level of SVD, a mathematical justification is conducted. In $[21,22]$ it is shown that blocking in the cover image increases the robustness of the watermark.

The mathematical proof in this section consists of one deduction which can be divided into two parts.

The first part proves that S coefficients in SVD decomposition are more stable when blocking is performed on the cover in comparison to doing SVD without blocking, and the second part proves that performing SVD on each block of our first deduction and gathering $S(1,1)$ in a separate matrix and performing SVD for this new matrix increases the stability of $S$ coefficients in the second SVD. Stability shows that when exposing the image to attacks, the values of $S(1,1)$ coefficients are less changed and are not substantially modified. As a result, the S coefficients in the second level of SVD are more robust against small perturbations in comparison to the $S$ coefficients when only one level of SVD is performed on the host image.

Deduction: in this deduction we first consider $\mathrm{A}$ as an image matrix. After decomposing $A$ to singular value decomposition (SVD), we have

$$
A=U S V^{T}
$$

If the watermark is hidden on S coefficients then we have

$$
A^{*}=U S^{*} V^{T}
$$


where $\mathrm{A} *$ is called watermarked picture and $\mathrm{U}$ and $\mathrm{V}$ are singular vectors of image A. The watermark is hidden in $S$ coefficients based on the following formula:

$$
S^{*}=S+\alpha S^{\prime}
$$

where $S$ is the singular values of the decomposition matrix of image $A$, and $S^{\prime}$ is the singular values of decomposition matrix of watermark $\mathrm{W}$, and $\alpha$ is the scaling factor.

Instead of $S *$ in (5), (6) is replaced to get

$$
A^{*}=U\left(S+\alpha S^{\prime}\right) V^{T}
$$

This is equal to

$$
\begin{aligned}
& A^{*}=U S V^{T}+U \alpha S^{\prime} V^{T} \Longrightarrow \\
& A^{*}=A+U \alpha S^{\prime} V^{T}
\end{aligned}
$$

On the other hand, $\left\langle\alpha \mathrm{S}^{\prime}\right\rangle$ is regarded as $\Delta \mathrm{S}$, which means the variation of the $S$ coefficients of the image after addition of the watermark. In respect to imperceptibility, $\alpha \mathrm{S}^{\prime}$ or $\Delta S$ should be as small as possible. For this purpose, the scaling factor " $\alpha$ " is multiplied by the $S$ coefficients of the watermark. If these variations are very small, this means that the limitation of (8) when $\Delta s \longrightarrow 0$ will be a very small value like $\varepsilon$, but not zero. Thus, we have

$$
\begin{aligned}
A^{*} & =A+U \boldsymbol{\alpha} S^{\prime} V^{T}=A+U \Delta S V^{T} \Longrightarrow \\
\lim _{\Delta S \longrightarrow 0}\left(A+U \Delta S V^{T}\right) & =A+\boldsymbol{\varepsilon} A \Longrightarrow \\
A^{*} & =A+\boldsymbol{\varepsilon} A
\end{aligned}
$$

On the other hand, if image $\mathrm{A}$ is divided into four blocks $\mathrm{A} 1$ and $\mathrm{A} 2, \mathrm{~A} 3$, and $\mathrm{A} 4$, such that $A=A_{1}+A_{2}+A_{3}+A_{4}$ :

$$
A=\left[\begin{array}{cc}
A 1 & A 2 \\
A 3 & A 4
\end{array}\right] \Longrightarrow \begin{cases}S V D & A 1=U_{A 1} S_{A 1} V_{A 1}^{T} \\
S V D & A 2=U_{A 2} S_{A 2} V_{A 2}^{T} \\
S V D & A 3=U_{A 3} S_{A 3} V_{A 3}^{T} \\
S V D & A 4=U_{A 4} S_{A 4} V_{A 4}^{T}\end{cases}
$$

Then, gathering all S(1,1) of each SA1, SA2, SA3, and SA4 into another matrix called $\mathrm{B}$ and decomposing matrix $\mathrm{B}$ into its singular values, we have

$$
B=\left[\begin{array}{ll}
S A 1(1,1) & S A 2(1,1) \\
S A 3(1,1) & S A 4(1,1)
\end{array}\right]
$$

After SVD since $\operatorname{SVD}(B)=U_{B} S_{B} V_{B}^{T}$ after hiding the watermark, $\mathrm{B}$ is changed to $\mathrm{B} *$ and decomposition of matrix $\mathrm{B} *$ is

$$
\begin{gathered}
B^{*}=B+\Delta B \Longrightarrow \\
\operatorname{SVD}\left(B^{*}\right)=\operatorname{SVD}(B+\Delta B)
\end{gathered}
$$

Since the watermark is hidden in singular values or SB coefficients, $\operatorname{SVD}\left(B^{*}\right)=\operatorname{SVD}(B+\Delta B)$, SVD can be written as $U_{B}\left(S_{B}+\alpha S_{B}^{\prime}\right) V_{B}^{T}$, so (12) can be written as

$$
\begin{aligned}
\operatorname{SVD}\left(B^{*}\right) & =\operatorname{SVD}(B+\Delta B)=U_{B}\left(S_{B}+\alpha S_{B}^{\prime}\right) V_{B}^{T} \\
& =U_{B}\left(S_{B}\right) V_{B}^{T}+U_{B}\left(\alpha S_{B}^{\prime}\right) V_{B}^{T} \\
& =B+U_{B}\left(\alpha S_{B}^{\prime}\right) V_{B}^{T} \Longrightarrow \\
\operatorname{SVD}(B *) & =B+U_{B}\left(\alpha S_{B}^{\prime}\right) V_{B}^{T}
\end{aligned}
$$

Since $\alpha S_{B}^{\prime}=\Delta S_{B}$, (13) is written as

$\operatorname{SVD}\left(B^{*}\right)=B+U_{B}\left(\Delta S_{B}\right) V_{B}^{T}$ When the changes of $\Delta S_{B} \longrightarrow$ 0 , it means that $\Delta S_{B}$ would be very small value like $\varepsilon_{B}$. In the following it is expressed mathematically:

$$
\begin{aligned}
& B^{*}=B+U_{B}\left(\alpha S_{B}^{\prime}\right) V_{B}^{T}=B+U_{B}\left(\Delta S_{B}\right) V_{B}^{T} \Longrightarrow \\
& \lim _{\Delta S_{\mathrm{B}} \longrightarrow 0}\left(\mathrm{~B}+\mathrm{U}_{\mathrm{B}} \Delta \mathrm{S}_{\mathrm{B}} \mathrm{V}_{\mathrm{B}}^{\mathrm{T}}\right)=\mathrm{B}+\varepsilon \mathrm{B} \Longrightarrow \\
& B *=B+\varepsilon B
\end{aligned}
$$

Compare (9) to (14), since $B \subseteq A$ and $B \in \bigcup_{i=1}^{4} S_{(1,1)}^{A i} \Longrightarrow$ Variation of $\mathrm{B}<$ variation $\mathrm{A}$.

Since both $S_{A}$ and $S_{B}$ qualities are from $S$ coefficients or in other words, $S_{A}$ is made of singular values of $A$, and $S B$ is made of $S(1,1)$ second singular values of $A$, so the variation and sensitivity of $S$ coefficients are very small against perturbation of image processing and geometric attacks based on [23]. Since in (14), it was proved that variation of $S_{B}$ is less than $S_{A}$ and $S_{A}$ is stable against small perturbation, it is believed that the variation of $S_{B}$ is less than of $S_{A}$. As a result $S_{B}$ is more robust against signal processing and geometric attacks in comparison to $\mathrm{S}_{\mathrm{A}}$.

In this deduction it is proved that the variations and constraints of the B matrix is less than variations and constraints in the A matrix.

Hence, inserting the watermark in $\mathrm{B}$ matrix, including high energetic parts of $S$ coefficients of matrix of $A$, after dividing it into blocks, is more robust than inserting the watermark in only the singular values of $\mathrm{A}$. The reason is that since the amount of the watermark that should be hidden in both $\mathrm{A}$ and $\mathrm{B}$ is the same, and because $\mathrm{B}<\mathrm{A}$, the $\mathrm{B}$ coefficients are more decomposed in comparison to A coefficients in SVD. Then after inverse SVD (ISVD) for matrix of $A$, we only have $U_{A}\left(S_{A}+\Delta S_{A}\right) V_{A}^{T}$, while for $B$ we have $U_{A}\left(U_{B}\right.$ $\left.\left(\mathrm{S}_{\mathrm{B}}+\Delta \mathrm{S}_{\mathrm{B}}\right) \mathrm{V}^{\mathrm{T}}{ }_{\mathrm{B}}\right) \mathrm{V}_{\mathrm{A}}{ }^{\mathrm{T}}$. Hence the watermark is more scattered in $\mathrm{B}$ in comparison to $\mathrm{A}$, and scattering will increase the robustness.

This deduction can be extended for more than four blocks in blocking of the cover image. Later on, we will also prove this again based on experimental results.

4.1. Inherited Specification of Singular Values in SVD2. After SVD transformation, the singular values have the specific characteristics which make them consistent with some geometric and signal processing distortions as follows $[19,20]$ : 
(i) Transpose Invariance: Matrix A and its transpose $\mathrm{A}^{\mathrm{T}}$ have the same nonzero singular values.

(ii) Flip Invariance: Matrix $A$, the row flip $A_{r f}$, and the column flip $\mathrm{A}_{\mathrm{cf}}$ have the same nonzero singular values.

(iii) Rotation Invariance: Matrix $\mathrm{A}$ and $\mathrm{A}_{\mathrm{r}}$ (A rotated by an arbitrary angle) have the same nonzero singular values.

(iv) Scale Invariance: If we scale up A by $\mathrm{L}_{1}$ times in row and by $\mathrm{L}_{2}$ times in column simultaneously, for every nonzero singular value $\lambda$ of $\mathrm{A} \sqrt{L_{1} L_{2} \lambda}$ is a nonzero singular value of the scaled up image. The two images will have the same number of nonzero singular values.

(v) Translation Invariance: If $\mathrm{A}$ is expanded by adding rows and columns of black pixels, the resulting matrix $A_{e}$ has the same nonzero singular values as A.

(vi) Transpose Invariance: Matrix A and its transpose $\mathrm{A}^{\mathrm{T}}$ of the second level of SVD have the same non-zero singular values.

Since SVD2 has the same quality of SVD1, all the specifications of SVD2 are inherited from SVD1. Here, the Transpose Invariance specification is proved and the other specifications are referenced to [23].

Now, we prove that the B matrix which made up of $S$ (1, 1) of each $n * n$ blocks of $A$ inherits the transpose invariance specification from A matrix. In other words, the Transpose Invariance specification from the A matrix can be moved to the B matrix.

Proof. Consider matrix A and decompose it to its singular value and vectors. So we have

$$
A=U S V^{T}
$$

If we decompose its singular values into second SVD then (15) is changed to

$$
A=U\left(U_{B} S_{B} V_{B}^{T}\right) V^{T}
$$

Then transposing matrix A will be concluded to

$$
\begin{aligned}
& A^{T}=\left[U\left(U_{B} S_{B} V^{T}{ }_{B}\right) V^{T}\right]^{T} \Rightarrow \\
& A^{T}=\left(V^{T}\right)^{T}\left(U_{B} S_{B} V^{T}{ }_{B}\right)^{T} U^{T}=> \\
& A^{T}=V\left(U_{B} S_{B} V^{T}{ }_{B}\right)^{T} U^{T}
\end{aligned}
$$

Then

$$
\mathrm{A}^{\mathrm{T}}=\mathrm{V}\left[\left(\mathrm{V}^{\mathrm{T}}{ }_{\mathrm{B}}\right)^{\mathrm{T}} \mathrm{S}_{\mathrm{B}}{ }^{\mathrm{T}} \mathrm{U}_{\mathrm{B}}{ }^{\mathrm{T}}\right] \mathrm{U}^{\mathrm{T}}=\mathrm{V}\left(\mathrm{V}_{\mathrm{B}} \mathrm{S}_{\mathrm{B}}{ }^{\mathrm{T}} \mathrm{U}_{\mathrm{B}}{ }^{\mathrm{T}}\right) \mathrm{U}^{\mathrm{T}}
$$

Since $S_{B}$ is a diagonal matrix, in every diagonal matrix, both matrix and its transpose are the same. Then, $S_{B}{ }^{T}=S_{B}$ and (18) can be written a:

$$
\mathrm{A}^{\mathrm{T}}=\mathrm{V}\left(\mathrm{V}_{\mathrm{B}} \mathrm{S}_{\mathrm{B}} \mathrm{U}_{\mathrm{B}}^{\mathrm{T}}\right) \mathrm{U}^{\mathrm{T}}
$$

But considering (15) and (16) the statement in the parenthesis $\left(V_{B} S_{B} U_{B}^{T}\right)$ is the same definition for $S^{T}$.
Hence, (19) can be written as

$$
\begin{aligned}
A^{T} & =V S^{T} U^{T} \\
\text { or } A^{T} & =\left(U S V^{T}\right)^{T}
\end{aligned}
$$

Thus, it is proved that singular values in B matrix are also transpose invariant.

All mentioned specifications of $\mathrm{S}$ coefficients are extensible and can be applied on $\mathrm{S}$ coefficients of $\mathrm{B}$ matrix due to identical quality of $\mathrm{S}$ coefficients in both $\mathrm{A}$ and $\mathrm{B}$ matrix.

\section{Experimental Results of the Host Feasibility Deduction}

This section is dealing with the experimental deduction. It is in fact the results of a feasibility test to prove the selected points for inserting the watermark, which are that "S" coefficients of the second level of SVD are suitable and robust to embed the watermark and their robustness is more than "S" coefficients in the first level of SVD.

After proving the idea of enhancing the robustness (by using the second level of SVD), theoretically and mathematically, an experimental test is ordered. For this purpose 60 pictures from two data bases of medical and normal images are exposed on the most predominant geometric and signal processing attacks to experimentally demonstrate that the " $S$ " coefficients in the second level of SVD are more robust than " $\mathrm{S}$ " coefficients in the first level.

Since the hosts images are selected from two databases, all images which were selected from "http://sipi.usc.edu/ database" are called normal images and all images chosen from "http://radiopedia.org/encyclopesia/cases/all" database are referred to medical images.

60 host images are exposed to different types of geometric and signal processing attacks, and based on the performance metric mentioned in Equation (21), the cover images are examined. Images types are including both medical and normal images. The examined attacks are Gaussian noise 0.01 , Gamma Correction 0.1, Average filter $3 \times 3$, Crop 1/2, Salt and Pepper 0.01, Scaling 1/2, Speckle 0.01, Median filtering $3 \times 3$, and Rotation 50. Normalized Correlation Coefficient NC is calculated for the " $\mathrm{S}$ " coefficients in the first and second level of SVD.

The main reason behind this comparison is to understand how much the "S" coefficients are similar before and after attacks. Thus, once SVD in the first level is performed on the image, the NC is checked for the first level of SVD by the following formula:

$$
N C 1=\frac{\sum_{M=1} \sum_{N=1} S_{S V D 1} \times S_{S V D 1}{ }^{*}}{\sqrt{\sum_{M=1} \sum_{N=1} S_{S V D 1}^{2}}}
$$

where $S_{S V D 1}$ is the first level of $S$ coefficients in SVD host image before attack and $S_{S V D 1}{ }^{*}$ is the first level of $S$ coefficients in SVD host image after attacks. By means of this, the stability of $S$ components in the host image at the first level of SVD decomposition is investigated. 


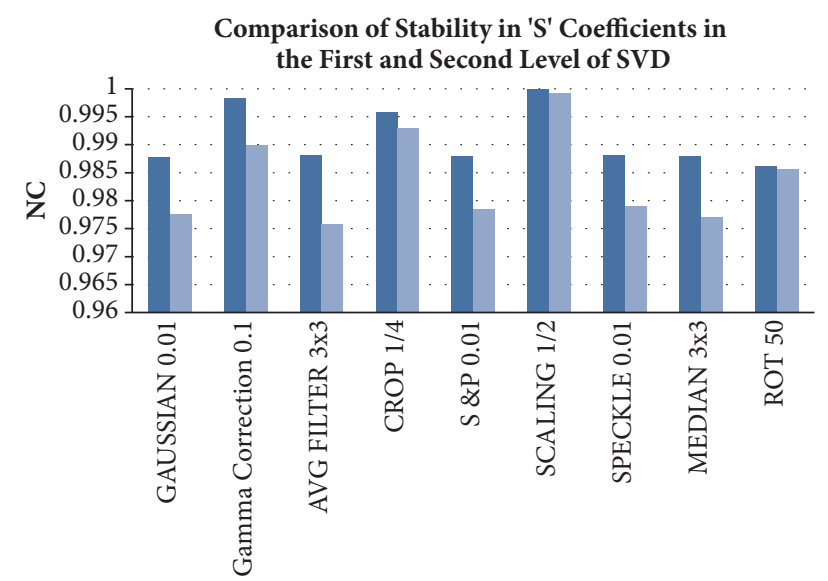

Attacks

- SVD 2

SVD 1

FIGURE 5: Comparison of stability in "S" coefficients in the first and second level of SVD for 33 images $512 * 512$.

For the second time the NC will be calculated by the following formula, but for the second level of SVD again in host image.

$$
N C 2=\frac{\sum_{M=1} \sum_{N=1} S_{S V D 2} \times S_{S V D 2}{ }^{*}}{\sqrt{\sum_{M=1} \sum_{N=1} S_{S V D 2}{ }^{2}}}
$$

In order to prove the stability and resistance of "S" coefficients in the second level of SVD, the NC1 resulted from comparison of " $\mathrm{S}$ " coefficients in first level will be compared to NC2 resulted from comparison of "S" coefficients in the second level. The larger NC shows the more stability and ability to resist against attacks.

The test is performed on different image sizes from both medical and normal images.

In the following, the average $\mathrm{NC}$ for the first and second level of SVD among 33 images with the size of $512 * 512,9$ images of $1024 * 1024$, and 18 images of $256 * 256$ are compared. The experimental results are shown in Tables 1-3.

As shown in Figure 5 and Table 1, it is clear that the "S" coefficients in the second level of SVD are more stable and their levels of resistance against attacks are better than "S" coefficients in the first level. This experiment is repeated for different sizes of images such as $1024 * 1024$ and $256 * 256$, and all the results confirm this finding.

Figure 6 and Table 2 illustrate the average NC for the "S" coefficients in the first and second level of SVD for several normal and medical images with the size of $1024 * 1024$. As shown in Figure 6, in all of the attacks, NC for SVD 2 is more than NC in SVD 1. SVD 2 stands for "S" coefficients in the second level of SVD, while SVD 1 represents "S" coefficients in the first level of SVD. The same results as the previous experiment demonstrate the level of resistance of "S" coefficients in the second level of SVD against the attacks and superiority of these coefficients in comparison to the "S" coefficients in the first level of SVD.

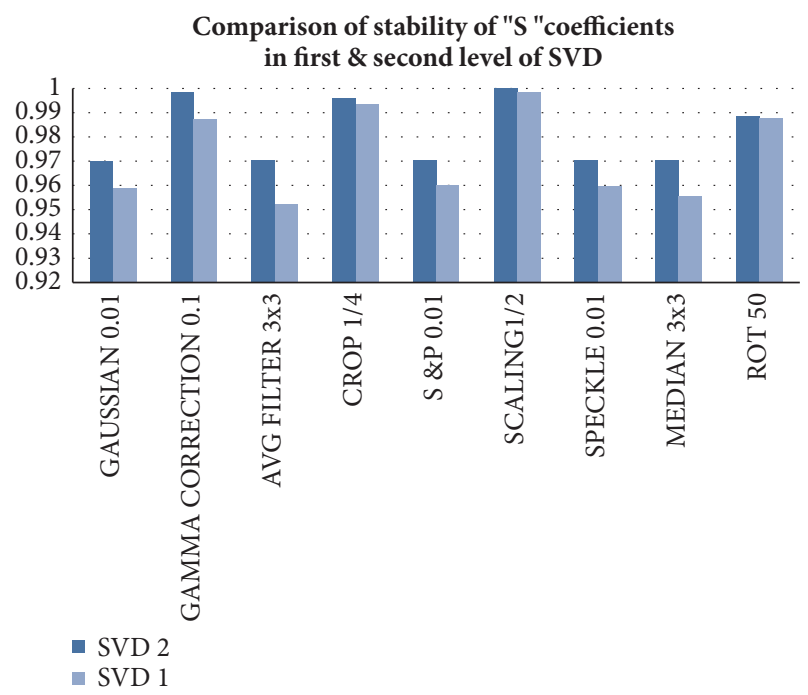

FIGURE 6: Comparison of stability in "S" coefficients in the first and second level of SVD for 9 images $1024 * 1024$.

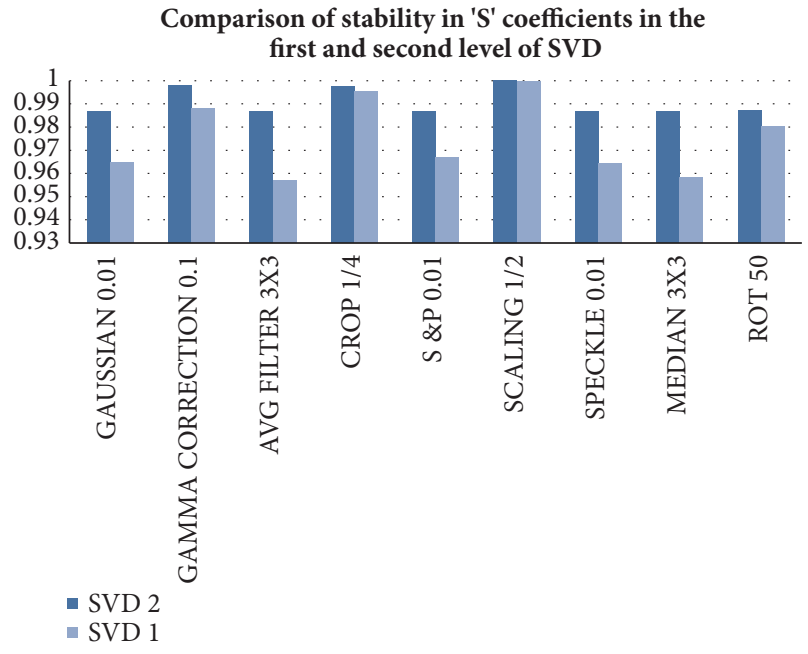

FIGURE 7: Comparison of stability in "S" coefficients in the first and second level of SVD for 18 images $256 * 256$.

As represented in Figure 7 and Table 3, in all of the attacks, NC for SVD 2 is more than NC in SVD 1. SVD 2 stands for " $S$ " coefficients in the second level of SVD, while SVD 1 represents "S" coefficients in the first level of SVD. Regarding this experiment, the stability and superiority of "S" coefficients in the second level of SVD are totally demonstrated in various types and size of images. As a result, these coefficients are considered as a good host for image hiding. In this experiment, all normal images are taken from USC-SIPI image database with the address http://sipi .usc.edu/database, and all medical images are taken from http://radiopedia.org/encyclopesia/cases/all radiology cases including the real samples of patients. This database has a variety of samples of real medical cases with the different modalities of MRI, CT, and X-RAY. 
TABLE 1: Average NC for S coefficients in the first and second level of SVD for 33 images $512 * 512$.

\begin{tabular}{lccccccccc}
\hline Attacks & $\begin{array}{c}\text { Gaussian } \\
\mathbf{0 . 0 1}\end{array}$ & $\begin{array}{c}\text { Gamma } \\
\text { correction } \mathbf{0 . 1}\end{array}$ & $\begin{array}{c}\text { AVG filter } \\
\mathbf{3} * \mathbf{3}\end{array}$ & Crop 1/4 & $\begin{array}{c}\text { S\&P } \\
\mathbf{0 . 0 1}\end{array}$ & Scaling 1/2 & $\begin{array}{c}\text { Speckle } \\
\mathbf{0 . 0 1}\end{array}$ & $\begin{array}{c}\text { Median } \\
\mathbf{3} * \mathbf{3}\end{array}$ & Rot 50 \\
\hline $\mathrm{NC}_{\mathrm{SVD} 2}$ & 0.9877 & 0.9983 & 0.9881 & 0.9959 & 0.9880 & 1 & 0.9882 & 0.9880 & 0.9861 \\
$\mathrm{NC}_{\mathrm{SVD} 1}$ & 0.9775 & 0.9899 & 0.9757 & 0.9929 & 0.9785 & 0.9992 & 0.9790 & 0.9771 & 0.9857 \\
\hline
\end{tabular}

TABLE 2: Average of NC for "S" coefficients in first and second level of SVD for 9 images $1024 * 1024$.

\begin{tabular}{lccccccccc}
\hline \multirow{2}{*}{ Attacks } & $\begin{array}{c}\text { Gaussian } \\
\mathbf{0 . 0 1}\end{array}$ & $\begin{array}{c}\text { Gamma } \\
\text { correction } \mathbf{0 . 1}\end{array}$ & $\begin{array}{c}\text { AVG filter } \\
\mathbf{3} * \mathbf{3}\end{array}$ & Crop 1/4 & $\begin{array}{c}\text { S\&P } \\
\mathbf{0 . 0 1}\end{array}$ & Scaling 1/2 & $\begin{array}{c}\text { Speckle } \\
\mathbf{0 . 0 1}\end{array}$ & $\begin{array}{c}\text { Median } \\
\mathbf{3} * \mathbf{3}\end{array}$ & Rot 50 \\
\hline $\mathrm{NC}_{\text {SVD2 }}$ & 0.9702 & 0.9986 & 0.9704 & 0.9959 & 0.9704 & 1 & 0.9705 & 0.9703 & 0.9887 \\
$\mathrm{NC}_{\text {SVD1 }}$ & 0.9587 & 0.9875 & 0.9522 & 0.9935 & 0.9602 & 0.9984 & 0.9596 & 0.9555 & 0.9878 \\
\hline
\end{tabular}

TABLE 3: Average of NC for "S" coefficients in first and second level of SVD for 18 images $256 * 256$.

\begin{tabular}{|c|c|c|c|c|c|c|c|c|c|}
\hline Attacks & $\begin{array}{c}\text { Gaussian } \\
0.01\end{array}$ & $\begin{array}{c}\text { Gamma } \\
\text { correction } 0.1\end{array}$ & $\begin{array}{c}\text { AVG filter } \\
\quad 3 * 3\end{array}$ & Crop 1/4 & $\begin{array}{c}\text { S\&P } \\
0.01\end{array}$ & Scaling $1 / 2$ & $\begin{array}{c}\text { Speckle } \\
0.01\end{array}$ & $\begin{array}{c}\text { Median } \\
3 * 3\end{array}$ & Rot 50 \\
\hline $\mathrm{NC}_{\mathrm{SVD} 2}$ & 0.9968 & 0.9980 & 0.9866 & 0.9976 & 0.9868 & 1 & 0.9867 & 0.9865 & 0.9871 \\
\hline $\mathrm{NC}_{\text {SVD1 }}$ & 0.9646 & 0.9878 & 0.9569 & 0.9952 & 0.9668 & 0.9994 & 0.9644 & 0.9580 & 0.9804 \\
\hline
\end{tabular}

\section{Conclusion and Future Work}

In this paper no specific implementation is presented. The aim is to ensure that our investigations tend to a successful implementation without wasting time, money and resources. The focus is to find regions of interest on the host image which are stable points for image watermarking such that least alteration is shown when they faced with signal processing and geometric distortions. For this purpose, firstly, basic theories to enhance the robustness are highlighted and then they are proved mathematically. Results of this theoretical and mathematical study ended up to use the second level of SVD to increase stability of the region of interest to embed watermarks. More stability leads to more robustness. After mathematical proof, it is necessary to show the robustness of "S" coefficients in the second level of SVD experimentally. For this purpose, an experiment was conducted on various host images before embedding the watermark. In this experiment, 60 host normal and medical images were exposed on geometric and signal processing attacks and the stability of "S" coefficients in the first and second level of SVD are compared. The experimental results proved the superiority of "S" coefficients in the second level of SVD in comparison to the first level in terms of robustness. Future work is to develop an image watermarking scheme based on the second level of SVD and to devise an authentication system in order to omit the false positive detection due to use "S" coefficients in SVD.

\section{Data Availability}

The data used to support the findings of this study are available from the corresponding author upon request.

\section{Conflicts of Interest}

The authors declare that they have no conflicts of interest.

\section{References}

[1] M. Rahman, "A DWT, DCT and SVD based watermarking technique to protect the image piracy," International Journal of Managing Public Sector Information \& Communication Technologies, vol. 4, 2013.

[2] S. Rohith and K. Bhat, "A Simple Robust Digital Image Watermarking against Salt and Pepper Noise using Repetition Codes," International Journal on Signal \& Image Processing, vol. 3, 2012.

[3] D. Arya, "A survey of frequency and wavelet domain digital watermarking techniques," International Journal of Scientific ¿amp; Engineering Research, vol. 1, 2010.

[4] X.-C. Yuan and C.-M. Pun, "Feature extraction and local Zernike moments based geometric invariant watermarking," Multimedia Tools and Applications, pp. 1-23, 2013.

[5] T. K. Araghi, M. Zamani, A. B. T. AbdulManaf, and S. K. Araghi, "A Survey on Digital Image Watermarking Techniques in Spatial and Transform Domains," in Proceedings of the International Journal of Advances in Image Processing Techniques- IJIPT, vol. 3, pp. 6-10, 2016.

[6] T. K. Araghi and A. B. A. Manaf, "Evaluation of Digital Image Watermarking Techniques," in Proceedings of the International Conference of Reliable Information and Communication Technology, pp. 361-368, 2017.

[7] N. M. Makbol and B. E. Khoo, "Robust blind image watermarking scheme based on redundant discrete wavelet transform and singular value decomposition," AEÜ - International Journal of Electronics and Communications, vol. 67, no. 2, pp. 102-112, 2013.

[8] M. Ali and C. W. Ahn, "An optimized watermarking technique based on self-adaptive DE in DWT-SVD transform domain," Signal Processing, vol. 94, no. 1, pp. 545-556, 2014.

[9] N. M. Makbol, B. E. Khoo, T. H. Rassem, and K. Loukhaoukha, "A new reliable optimized image watermarking scheme based on the integer wavelet transform and singular value decomposition for copyright protection," Information Sciences, vol. 417, pp. 381-400, 2017.

[10] S. Ojha, A. Sharma, and R. Chaturvedi, "Centric-Oriented Novel Image Watermarking Technique Based on DWT and 
SVD," in Soft Computing: Theories and Applications, vol. 583 of Advances in Intelligent Systems and Computing, pp. 217-225, Springer, 2018.

[11] I. A. Ansari and M. Pant, "Multipurpose image watermarking in the domain of DWT based on SVD and ABC," Pattern Recognition Letters, vol. 94, pp. 228-236, 2017.

[12] T. K. Araghi, A. A. Manaf, and S. K. Araghi, "A secure blind discrete wavelet transform based watermarking scheme using two-level singular value decomposition," Expert Systems with Applications, vol. 112, pp. 208-228, 2018.

[13] P. Lipiński, "On domain selection for additive, blind image watermarking," Bulletin of the Polish Academy of Sciences-Technical Sciences, vol. 60, no. 2, pp. 317-321, 2012.

[14] A. Kumar Gupta and M. S. Raval, "A robust and secure watermarking scheme based on singular values replacement," SADHANA - Academy Proceedings in Engineering Sciences, vol. 37, no. 4, pp. 425-440, 2012.

[15] M. Agoyi, E. Çelebi, and G. Anbarjafari, "A watermarking algorithm based on chirp z-transform, discrete wavelet transform, and singular value decomposition," Signal, Image and Video Processing, vol. 9, no. 3, pp. 735-745, 2014.

[16] W.-H. Kim, H.-U. Jang, J.-U. Hou, and H.-K. Lee, Robust Template-Based Watermarking for DIBR 3D Images, 2018.

[17] T. K. Araghi, B. T. Azizah, S. Kohpayeh, M. Zamani et al., "Taxonomy and Performance Evaluation of Feature Based Extraction Techniques in Digital Image Watermarking," International Journal Of Image Processing Techniques- IJIPT, vol. 3, pp. 20-23, 2016.

[18] R. Reddy, D. S. Rao, and M. V. N. K. Prasad, "Robust digital watermarking of color images under noise attacks," International Journal of Recent Trends in Engineering, vol. 1, pp. 334$338,2009$.

[19] V. Aslantas, "An optimal robust digital image watermarking based on SVD using differential evolution algorithm," Optics Communications, vol. 282, no. 5, pp. 769-777, 2009.

[20] S. Rawat and B. Raman, "Best tree wavelet packet transform based copyright protection scheme for digital images," Optics Communications, vol. 285, no. 10-11, pp. 2563-2574, 2012.

[21] J. Aparna and S. Ayyappan, "Image Watermarking Using Diffie Hellman Key Exchange Algorithm," Procedia Computer Science, vol. 46, pp. 1684-1691, 2015.

[22] R. A. Ghazy, A. M. Abbas, N. Al-Zubi et al., "Block-based SVD image watermarking in spatial and transform domains," International Journal of Electronics, vol. 102, no. 7, pp. 1091-1113, 2015.

[23] B. Zhou and J. Chen, "A geometric distortion resilient image watermarking algorithm based on SVD," Journal of Image and Graphics, vol. 4, article 022, 2004. 


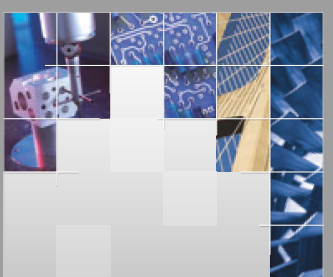

\section{Enfincering}
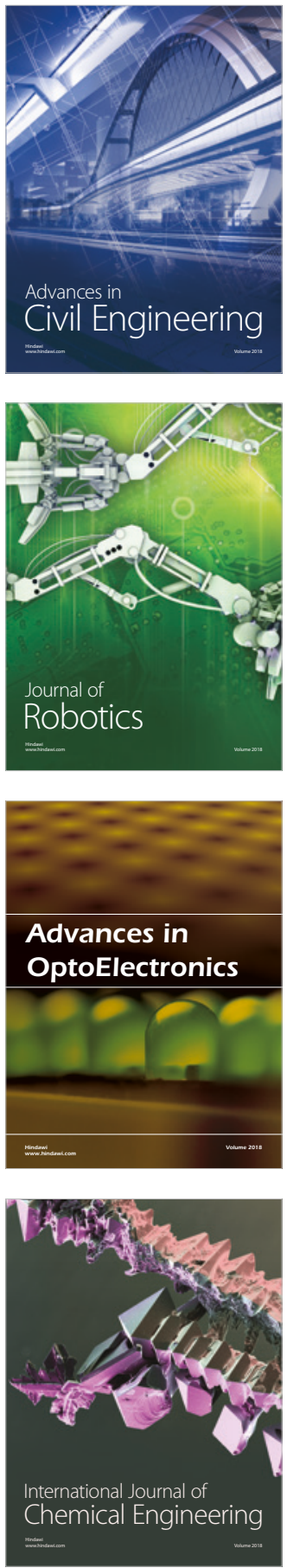

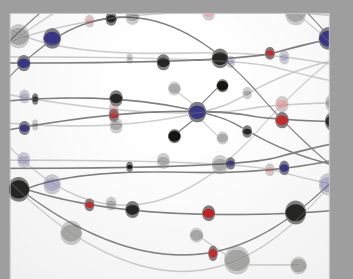

\section{Rotating \\ Machinery}

The Scientific World Journal

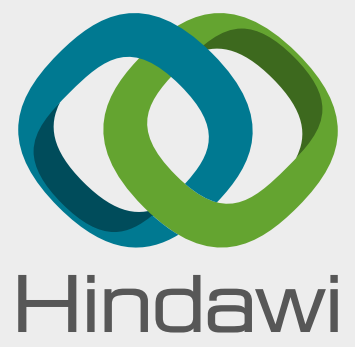

Submit your manuscripts at

www.hindawi.com
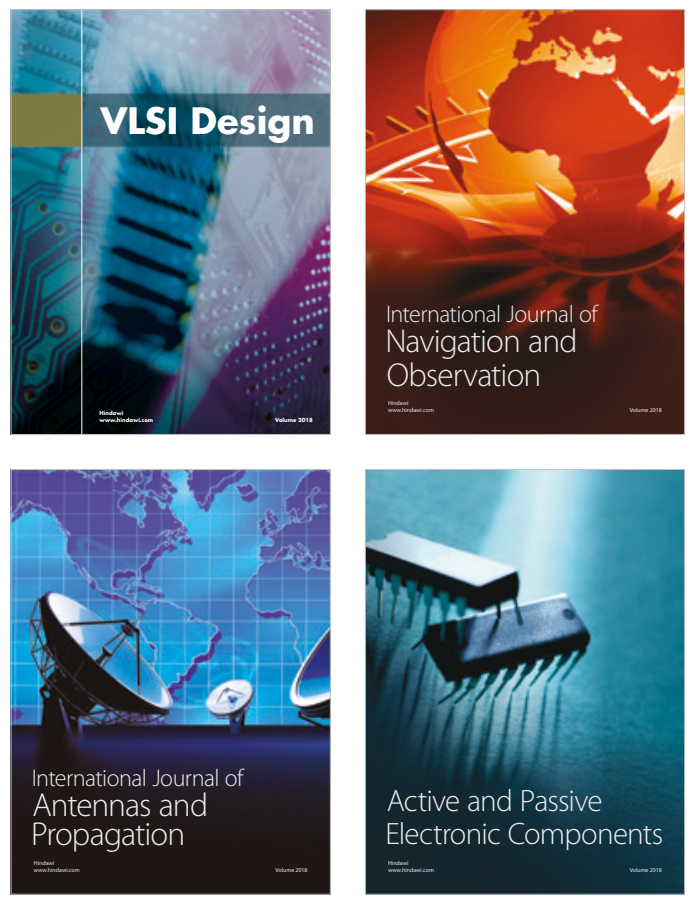
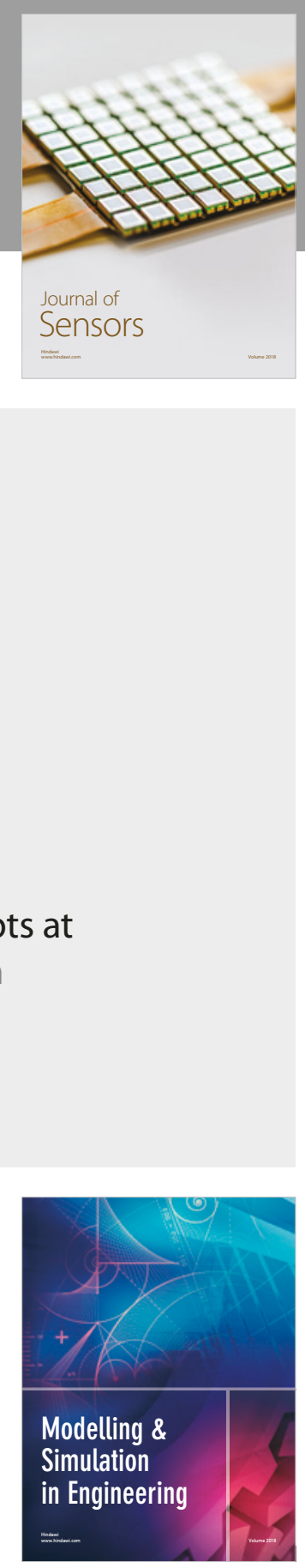

\section{Advances \\ Multimedia}
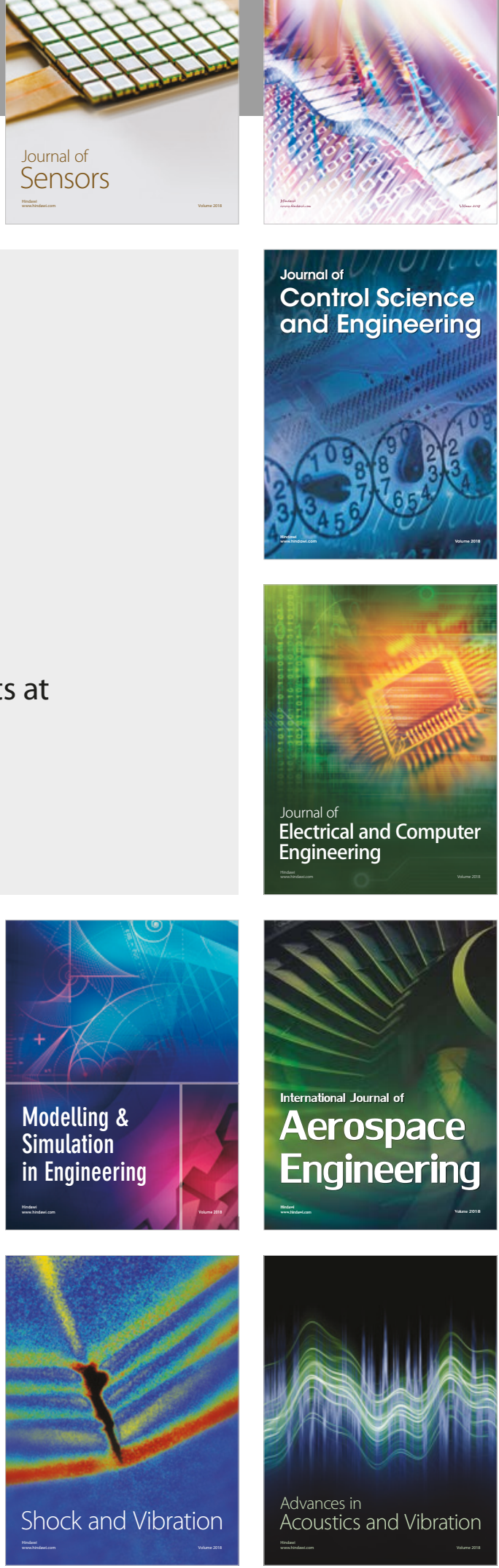